Journal of Social Sciences 6 (3): 411-415, 2010

ISSN 1549-3652

(C) 2010 Science Publications

\title{
Aggressive Driving Behavior: Undergraduate Students Study
}

\author{
Rungson Chomeya \\ Department of Educational Psychology and Guidance, Faculty of Education, \\ Mahasarakham University, Mahasarakham 44000, Thailand
}

\begin{abstract}
Problem statement: The main purposes of this research were (1) to study the aggressive driving behavior of graduate students, (2) to develop aggressive driving behavior standard test, (3) to compare the aggressive driving behavior between gender, years of study, academic achievement, driving confidence and driving experience and (4) to study the relationship among aggressive driving behavior, driving confidence, driving experience and accident experience. Approach: The subjects consisted of 471 undergraduate students who were selected by stratified random sampling method. The instrument was the aggressive driving behavior test, which discrimination of test was 2.00-6.56 and reliability was 0.85. Results: The research results revealed that (1) the mean score of the students on the aggressive driving behavior was at low level, (2) the test has the discrimination scores ranging from 8.34-20.82 and the reliability at 0.92 (3) there were no significant differences on aggressive driving behavior between years of study, academic achievement, driving confidence and driving experience; but the aggressive driving behavior showed significantly different between genders at 0.001 level and (4) the aggressive driving behavior had significant correlation coefficient at 0.05 level with driving confidence and driving experience and at 0.001 level with accident experience. All variables had low correlation coefficient. Conclusion/Recommendations: The study can be explained the causes of aggressive driving behavior occur on persons that how it has been developed in order to help understanding more.
\end{abstract}

Key words: Aggressive driving behavior, psychology test, undergraduate, Thailand

\section{INTRODUCTION}

Currently, using transportation, such as car, motorcycle, tricycle, bicycle or other vehicles, is a common way of life. The number of private vehicles has continuously increased. The reason is due to the economic growth, values and needs for convenient living. Due to the increasing number of vehicles, the public area has to be shared among drivers and that causes some drivers stressful. As a result, many drivers, while driving, have shown inappropriate behavior, especially aggression to others.

The aggressive behavior causes destruction and danger to persons and society as a whole. On the psychological point of view, aggressive behavior in terms of serious level is regarded as a kind of problem, because it results from the conditions which the persons cannot adjust themselves (Baker and Holzworth, 1961). The study of aggressive behavior helps researchers, educators and psychologists understand and be able to find the ways to adjust the behavior to become satisfying. The aggressive behavior is considered to be complex and involves both physical aggressive or verbal aggressive. On the view of Buddhism, the conceptual aggression is acceptable. In terms of psychology, the conceptual aggression is a covert behavior in both active and passive aspects. Therefore, the aggressive behavior has different levels of severity. The high level of severity can be dangerous to the society and lead to a crime, if a person cannot adjust or improve themselves or if he/she is in the situations where are proper to show their aggressive behavior.

Aggressive driving behavior is one of the aggressive behavior forms which is commonly found at the present time (Lajunen and Parker, 2004; Shinar and Compton, 2004; Galovski et al., 2005; Hohn, 2006). It may cause various problems in society, such as traffic jam, accident that affects the properties and life of people, illness and death. As we can see, the statistics of the dead from the accidents are increasing from the past years, although many organizations both governmental and private sections have been trying to prevent the accidents. According to a formal statistic of the death from New Year Festival accident, the death toll was raised up to 449 persons and wounded people were up to 4,943 persons (Department of Disaster Prevention and Mitigation, 2009). The causes of accidents resulted from several reasons; however, one of the reasons was drivers' aggressive driving behavior. 
The study of aggressive driving behavior is a new trend of physiological study and behavioral science in the USA and European countries. According to the documents and literature reviews, it is found that this study is still not found in Thailand and most of the research studies on driving are about accidents and traffic research. For that reason, the study of aggressive driving behavior is the initiation of development about this issue. The study is expected to contribute the knowledge base in terms of academy, concept and behavior measurement or behavior test (test standard development), research form development as well as research application in terms of concretely social development further.

\section{Objectives:}

- To study the aggressive driving behavior of undergraduate students

- To develop the aggressive driving behavior standard test

- To compare between different gender, years of study, learning achievement, driving confidence and driving experience on the aggressive driving behavior

- To study the relationship between the aggressive driving behavior and driving confidence, driving experience and person's accident experience

\section{Hypothesis:}

- There were differences between gender, years of study, learning achievement, driving confidence, driving experience and person's accident experience on aggressive driving behavior

- The aggressive driving behavior, driving confidence, driving experience and person's accident experience are related to each other

\section{MATERIALS AND METHODS}

Population was 28,275 Mahasarakham University undergraduate students from every faculty and major of study, in the semester 1 of 2008 academic year.

The samples for data collection were 450 undergraduate students, who enrolled in semester 1 of 2008 academic year. The samples size was determined based on the budgets of the study, funded by Mahasarakham University. The samples were the students who could drive motorcycle, tricycle or car by enquiring after finishing the random sampling. The independent variables included driving confidence, driving experience, accident experience and the dependent variables were the aggressive driving behavior and bi-social variables which were gender, class and learning achievement.

The researcher analyzed the data to test the research hypothesis according to the procedures. Mean and standard deviation for describing general information of the various variables of the research was used, t-test for considering the discrimination of the test instruments by dividing into the high and low group for analyzing with the technique 27 and 73 Percentiles and research hypothesis test, alpha co-efficient Value for considering the reliability of the test instrument, OneWay ANOVA for testing the research hypothesis and Pearson Product Moment Correlation were used for data analyzing.

\section{RESULTS}

The average score of aggressive driving behavior at the primary level was 73.32 and the standard deviation was 25.45. This means that the samples had the aggressive driving behavior at a low level. There were 409 persons who were polite drivers $(86.80 \%)$, whereas 1 person $(0.20 \%)$ showed a high risk to have a driving accident.

The aggressive driving behavior test had high discrimination. The t-test had statistical significance at the 0.001 level in every item. The whole test had the discrimination (t) ranging of 8.34-20.82 and the reliability was 0.92 .

When comparing the aggressive driving behavior between the male and female samples by using t-test analysis, the aggressive driving behavior was found to have a statistically significant difference at the 0.001 level. The male samples had the average score of aggressive driving behavior higher than female samples (males had the average score equal to 78.48 and females had the average score equal to 68.20), but there was no evidence of the differences between years of study, academic achievement, driving confidence and driving experience.

According to the relationship between aggressive driving behavior and driving confidence, driving experience and driving experience by the analysis of Pearson Product Moment Correlation, the aggressive driving behavior was found to have a positive relationship at a statistical significance level of 0.05 with the driving confidence and driving experience, but was at the low level. The aggressive driving behavior had a positive relationship with the driving experience at the statistical significance level of 0.001 , but was at the low level. 


\section{J. Social Sci., 6 (3): 411-415, 2010}

\section{DISCUSSION}

The study revealed that 471 samples had the average score of aggressive driving behavior at the primary level at 73.32 and the standard deviation at 25.45. This suggested that the samples had the aggressive driving behavior at the low level (409 persons or $86.80 \%$ ) and had the behavior of polite driving or had only little aggressive driving behavior, whereas one person $(0.20 \%)$ was found to have the aggressive driving behavior at the high level. The reason would be that the samples of the study were university students, who were much mature, comparing to other people at the same age who did not study. Also, the samples had learned and taught from their institutes how they improved themselves in various aspects.

The study revealed that every item of the aggressive driving behavior test had the discrimination at a high level. The t-test had the statistical significance at the 0.001 level and had the reliability of the tests at 0.92. It is obvious that there was the increasing of statistical value. The reason is because the development of aggressive driving behavior standard test was carefully constructed based on the concepts and theories related to such behavior and also was examined for it's validity of contents, construct validity, discrimination and reliability. Accordingly, the instrument was tried out with 50 undergraduate students prior to using with the samples and the result showed the acceptable discrimination ranging from 2.00-6.56 and reliability was 0.85 . This can be concluded that the test can be used for testing the aggressive driving behavior. When the researcher brought the test to use with 471 samples to confirm the quality of the instrument when using in the real situation with the large size of the samples, it was found that the discrimination was at the high level with the t-test between 8.34 and 20.82 whereas the reliability was equal to 0.92 . The reason is because in the process of the test development, the researcher carried out the various related concepts and theories together with the research results of aggressive driving behavior. The researcher drafted the test and then tried out for several times in order to get the correctness and appropriateness; therefore, this test can well examine the aggressive driving behavior. This is in accordance with the research of (Oetting, 2003) who studied the aggressive driving behavior by using the test called Driving Anger Situations: DAS. The test was determined the situations related to 14 driving situations and asked the respondents to answered the emotional report happened of themselves in terms of rating scale 5 points which were from 1 (none) to 5 (maximum). Also, in the research of Dukes et al. (2001) who made the aggressive driving behavior test by determining the various situations in each item related to the driving, the respondents reported the conditions of anger in terms of rating scale 7 points which had the level from 0-6.

The study result revealed that the aggressive driving behavior between male samples and female samples was different at statistical significance of the 0.001 level. The male samples had the average score of aggressive driving behavior higher than the female samples. That is because the gender is a significant factor to indicate the level of the aggressive behavior. Meechanchung (2001) viewed that the aggressive behavior was the crucial variable of definition on male and female. One reason is that males had the aggressive behavior because of their physical and biological components. Also, the aggressive behavior is resulted from the social learning, the society usually expected males to play the role different from females, for example, males were brave and strong and that affected males to present more severe and rugged behavior than females. This is in accordance with several documents which found that the males had the aggressive behavior more often, severe and longer than females (Trankkasombat, 1999).

When comparing the aggressive driving behavior with different years of study, learning achievement, driving confidence and driving experience, it was found that there was no significant difference. The reason is that the samples were not much different in their ages, their maturity in various aspects were not much different too. Based on psychological point of view, the maturity has an important impact on the emotional behavior control. Therefore, it makes the study results were not any different at all. This is in accordance with the research of (Dukes et al., 2001) studied the effects of aggressive driving behavior and driver's characteristics on driving. Their samples were 144 undergraduate students. The results revealed that the persons would presented their aggressive within different situations and found the relationship between aggressive driving behavior and effects happened to the driving but for the age which affected the driving, the result was not found.

When comparing between the samples with different learning achievements, it was found that the aggressive behavior was not different. The reason is that the learning achievement of the sample group was the academic competence of each individual, while the aggressive behavior was the continuous results from person's driving which relied on the competence together with private experiences of each individual. 
When comparing the aggressive behavior between the samples with different degrees of driving confidence, it was found that there was no difference by the statistical significance of 0.05 . That is because the driving confidence test in this study used the confidence level of the samples roughly with only 1 question. This made the results of variable evaluation lacked of refinement and could not be found the difference in terms of the issue. Similarly, when comparing the aggressive behavior between the samples with different driving experiences, it was found no difference. That is because the driving experience of the samples were similar and most of the samples $(74.90 \%)$ had the driving experience more than 5 years, therefore there was no difference in aggressive driving behavior.

The results of relationship between the aggressive driving behavior and the driving confidence, driving experience and accident experience with the analysis of Pearson Product Moment Correlation, it was found that the aggressive driving behavior had significant correlation coefficient at 0.05 level with driving confidence and driving experience and at 0.001 level with accident experience. All variables had low correlation coefficient. This confirms that the driving confidence and driving experience had the aggressive driving behavior. The reason is that the driving confidence test did not elaborate enough and this was the primary attributes survey. According to the driving experience, it was found the similarity whereas the aggressive driving behavior and the driving experience was only the primary survey because this study focused on studying and developing the aggressive driving behavior to be the main point.

\section{CONCLUSION}

- The aggressive driving behavior test developed in this research can be surveyed the aggressive driving behavior and this made the knowing of the behavior risks which may cause the effects in various aspects from vehicle use

- The data from the behavior test in each item measured the sub behaviors which had the details of behavior in several aspects which can be used for preventing and solving the problems occurred from the aggressive driving behavior, for example, the increasing of driving accident, quarrel from conflict due to the aggressive driving

- The researcher did not categorize between the car drivers and motorcycle drivers. However two types of driving were different in several conditions. Therefore, it should study about the aggressive driving behavior of car drivers or motorcycle drivers at the specific way to consider the difference of aggressive driving behavior of the two types of vehicles particularly

- It should study the aggressive driving behavior in terms of the finding factors related to the prediction of aggressive driving behavior explanation or finding the causes of aggressive driving behavior occur on persons that how it has been developed in order to help understanding about the aggressive driving behavior more

- It should have the survey study wide in the large size of the samples to see the level of aggressive driving behavior of general people or people who have different age and occupation to view the image of the aggressive driving behavior as a whole which will be beneficial for behavioral development plan or orient the ways to prevent the accident occurred from vehicles driving

\section{ACKNOWLEDGEMENT}

This research was financially supported by Mahasarakham University.

\section{REFERENCES}

Baker, J.W. and A. Holzworth, 1961. Social histories of successful and unsuccessful children. Child Dev., 32: 135-149.

Department of Disaster Prevention and Mitigation, 2009. Traffic Accident statistic. Fund for Health Promotion. http://www.thaihealth.or.th/node/11683

Dukes, R.L., S.L. Clayton, L.T. Jenkins, T.L. Miller and S.E. Rodgers, 2001. Effects of aggressive driving and driver characteristics on road rage. Soc. Sci. J., 38: 323-331. DOI: 10.1016/S03623319(01)00117-3

Galovski, T.E., L.S. Malta and E.B. Blanchard, 2005. Road Rage: Assessment and Treatment of the Angry, Aggressive Driver. 1st Edn., American Psychological Association, Washington DC., ISBN: 10: 1591473055, pp: 250.

Hohn, R.L., 2006. Aggressive driving and road rage: they aren't the same. PDE Publications Inc. http://www.drivers.com/article/462/

Lajunen, T. and D. Parker, 2001. Are aggressive people aggressive drivers? A study of the relationship between self-reported general aggressiveness, driver anger and aggressive driving. Accid. Anal. Prev., 33: 243-255. DOI: 10.1016/S00014575(00)00039-7 
Meechanchung, S., 2001. Some factors related to students aggressive behaviors in secondary schools under the Department of General Education in Buriram Province: Path analysis. Master Thesis, Mahasarakham University.

Oetting, E.R., 2003. Characteristics of two groups of angry drivers. J. Counsel. Psychol., 50: 123-132.
Shinar, D. and R. Compton, 2004. Aggressive driving: an observational study of driver, vehicle and situational variables. Accid. Anal. Prev., 36: 429-437. DOI: 10.1016/S0001-4575(03)00037-X

Trankkasombat, U., 1999. Discipline Your Child. 2nd Edn., Family Research and Development Center, Bangkok, ISBN: 13: 9748716546, pp: 260. 